\title{
Fructo-oligosaccharides purification from a fermentative broth using an activated charcoal column
}

\author{
C. Nobre, J.A. Teixeira and L.R. Rodrigues
}

IBB - Institute for Biotechnology and Bioengineering, Centre of Biological Engineering, University of Minho, Campus de Gualtar, 4710-057 Braga, Portugal

In this study, a simple and efficient process to purify fructo-oligosaccharides (FOS) from a fermentative broth was proposed using a single activated charcoal column. The FOS adsorption onto the activated charcoal was modeled by a pseudo-second order model. Several volumes and concentrations of water/ ethanol were studied to optimize the selective desorption of sugars from the broth mixture at $25{ }^{\circ} \mathrm{C}$. Mixtures containing $50.6 \%(\mathrm{w} / \mathrm{w}$ ) of FOS (FOS content in the fermentative broth) were purified to $92.9 \%$ $(\mathrm{w} / \mathrm{w})$ with a FOS recovery of $74.5 \%(\mathrm{w} / \mathrm{w})$. Moreover, with the proposed process, fractions with purity up to $97 \%(w / w)$ of FOS were obtained. This purification process was also found to be efficient in the desalting of the fermentative broth.

\section{Introduction}

Fructo-oligosaccharides (FOS) have gained large commercial interest due to their beneficial properties in the human health as prebiotics. FOS, namely, kestose $\left(\mathrm{GF}_{2}\right)$, nystose $\left(\mathrm{GF}_{3}\right)$ and fructofuranosylnystose $\left(\mathrm{GF}_{4}\right)$, are nondigestible food ingredients that are selectively fermented in the colon, increasing the number of beneficial bacteria by modulation of the gut microflora [1].

Although FOS occur naturally in many common foods such as fruit, vegetables, milk and honey, they are present in low concentrations and are season-limited. On a large scale FOS can be produced by fermentation using microorganisms, such as Aureobasidium sp. $[2,3]$. These fungi produce enzymes with transfructosylation activities that convert sucrose into FOS [4]. At the end of the process, the fermentative broth contains FOS together with microorganisms, salts and sugars with low molecular weight, such as fructose (F), glucose (G) and sucrose (S). These small sugars do not meet the requirements to be considered as prebiotics because they are metabolized by humans. Therefore, to obtain a mixture with high prebiotic activity these small sugars must be eliminated or reduced. Additionally, salts and microorganisms must also be removed.

Several methods have been developed to purify oligosaccharides from sugar mixtures [5], such as the use of ion exchange resins, activated charcoal, size exclusion chromatography, solvent pre-

Corresponding author: Nobre, C. (clarissenobre@deb.uminho.pt) cipitation, nanofiltration and yeast treatments [5-11]. Sanz and co-workers (2005) compared three of these methods - nanofiltration, yeast treatment and activated charcoal - regarding their potential to separate monosaccharides from oligosaccharides in honey. The authors found that activated charcoal was the most efficient method when a pure mixture in oligosaccharides is envisaged. Therefore, among other methods that have been studied, the adsorption onto activated charcoal persists as one of the most suitable methods. Activated charcoal is cheap and has a large surface area and pore volume, conducting to a good sorption capacity. In addition, this sorbent is easily regenerated [12] and has the potential to desalt solutions $[13,14]$. Other advantages consist in the simplicity of the system, the low cost of the process, and its capacity to separate large amounts of oligosaccharides using only a single small column $[14,15]$.

Since Whistler and Durso [14] reported in 1950 the use of activated charcoal in combination with Celite to separate sugars by elution with increasing ethanol concentrations, several researchers applied this method with different combinations of adsorbent and ethanol concentrations to purify sugars from honey [16-18]; oligosaccharides from human milk [19]; galacto-oligosaccharides from reaction mixtures [20,21]; and FOS from sugar mixtures $[10,22,23]$.

The isolated FOS $\left(\mathrm{GF}_{2}, \mathrm{GF}_{3}\right.$ and $\left.\mathrm{GF}_{4}\right)$ are only available for analytical purposes and their prices are prohibitive. Indeed, based 
on the information of the main suppliers of pure FOS (e.g. Sigma-Aldrich, Megazyme, Wako Chemicals $\mathrm{GmbH}$ ) conducted for the current work, it was found that even pure FOS mixtures are still very expensive. Hence, it is crucial to optimize the FOS purification processes for large-scale applications. In this study, a simple and efficient process to purify FOS from a fermentative broth using a single activated charcoal column is proposed and optimized. FOS were produced by fermentation of sucrose using Aureobasidium sp. The adsorption kinetics onto activated charcoal of the sugars contained in the fermentative broth was studied in shaken flasks and modeled using pseudo-first and pseudo-second order models. The purification process was conducted in three steps: (1) adsorption of sugars onto the activated charcoal column; (2) column washing with water; and (3) desorption of sugars with an ethanol gradient. Finally, the effectiveness of the activated charcoal on the mixture desalting was also evaluated.

\section{Materials and methods FOS production}

A $10 \mathrm{~mL}$ preculture of Aureobasidium sp. stored in glycerol was added to $75 \mathrm{~mL}$ of inoculation medium, in a shaken flask (inoculation medium: $100 \mathrm{~g} \mathrm{~L}^{-1}$ of sucrose, $10 \mathrm{~g} \mathrm{~L}^{-1}$ of $\mathrm{NaNO}_{3}, 0.5 \mathrm{~g} \mathrm{~L}^{-1}$ of $\mathrm{KCl}, 0.35 \mathrm{~g} \mathrm{~L}^{-1}$ of $\mathrm{K}_{2} \mathrm{SO}_{4}, 0.5 \mathrm{~g} \mathrm{~L}^{-1}$ of $\mathrm{MgSO}_{4} .7 \mathrm{H}_{2} \mathrm{O}, 7.89 \mathrm{~g} \mathrm{~L}^{-1}$ of $\mathrm{KH}_{2} \mathrm{PO}_{4}$ and $0.01 \mathrm{~g} \mathrm{~L}^{-1}$ of $\mathrm{FeSO}_{4} \cdot 7 \mathrm{H}_{2} \mathrm{O}$ ). The inoculum was grown for 3 days at $28{ }^{\circ} \mathrm{C}$ and $140 \mathrm{rpm}$ and transferred afterwards to a $5 \mathrm{~L}$ bioreactor - Model microDCU system, equipped with Twin and MCU-200 controllers (B. Braun Biotech International, Melsungen, Germany), using a working volume of $4 \mathrm{~L}$ of culture medium (culture medium: $200 \mathrm{~g} \mathrm{~L}^{-1}$ sucrose, $20 \mathrm{~g} \mathrm{~L}^{-1} \mathrm{NaNO}_{3}$ and the same salt concentrations as the ones used in the inoculum medium). Fermentations were carried out at $28{ }^{\circ} \mathrm{C}, 300 \mathrm{rpm}$ and $\mathrm{pH}$ 5.0. Several samples were taken at different points in time to evaluate their sugar profile. After detecting the production of the highest FOS molecule $\left(\mathrm{GF}_{4}\right)$ by High-Performance Liquid Chromatography (HPLC), the fermentation was stopped and cells were removed by filtration with a paper filter, followed by a microfiltration with a $0.2 \mu \mathrm{m}$ cut-off cellulose acetate filter. Residual proteins were removed by filtration with a centrifugal filter Amicon Ultra-15 $(50 \mathrm{NMWL})$ from Millipore, at $4000 \times g$ for $10 \mathrm{~min}$. Afterwards, the cell free fermentative broth was stored at $-18{ }^{\circ} \mathrm{C}$ for further purification. All chemicals used were of analytical grade.

The fermentative broth used in the column experiments had the following composition in mono- and disaccharides: $23.74 \mathrm{~g} \mathrm{~L}^{-1}$ of $\mathrm{F}, 58.34 \mathrm{~g} \mathrm{~L}^{-1}$ of $\mathrm{G}$ and $9.87 \mathrm{~g} \mathrm{~L}^{-1}$ of $\mathrm{S}$. Moreover, the FOS composition of the fermentative broth was: $41.88 \mathrm{~g} \mathrm{~L}^{-1}$ of $\mathrm{GF}_{2}$, $47.98 \mathrm{~g} \mathrm{~L}^{-1}$ of $\mathrm{GF}_{3}$ and $4.16 \mathrm{~g} \mathrm{~L}^{-1}$ of $\mathrm{GF}_{4}$. Hence, the initial percentage of FOS in total sugars was $50.6 \%(\mathrm{w} / \mathrm{w})$.

\section{Adsorbent}

The adsorbent used in all the purification experiments was activated charcoal extra pure, supplied by Merck (Darmstadt, Germany) in a granular form, with $1.5 \mathrm{~mm}$ of mean particle diameter.

\section{Adsorption kinetic studies}

According to the previous studies (data not shown), a volume of $35 \mathrm{~mL}$ of fermentative broth was added to $18 \mathrm{~g}$ of activated charcoal contained in a shaken flask. Adsorption was held for
5.5 hours, at $25^{\circ} \mathrm{C}$, and $140 \mathrm{rpm}$ of agitation. Samples were collected during the adsorption process and sugars from the liquid phase were analyzed by HPLC (see section 'Sugars analysis'). Experiments were carried out in duplicate.

The concentration of sugars adsorbed onto the resin, defined as the loading $(q)$, was determined according to the following equation:

$q=\frac{\left(C_{0}-C\right) \cdot V}{m}$

where $C_{0}$ and $C$ are the initial concentration and the concentrations at time $t$ or at the equilibrium, respectively, $V$ is the volume of solution and $m$ the dried activated charcoal mass.

\section{Activated charcoal column}

Before filling the column, activated charcoal was washed and autoclaved to remove particles and air from the pores. Next, $180 \mathrm{~g}$ of adsorbent was loaded into a column glass $(33 \mathrm{~cm} \times 4.5 \mathrm{~cm}$ $\mathrm{cm}$ internal diameter), previously filled with Milli-Q pure water. Milli-Q pure water was pumped from the bottom of the column using a peristaltic pump (Watson Marlow) to equilibrate the charcoal inside the column. To remove the fines, the pump needs to work at a higher flow-rate than the one which was used to purify the sugars $(25 \mathrm{~mL} / \mathrm{min})$, although this flow-rate should not be too high to prevent the charcoal elution. A flow-rate of $30 \mathrm{~mL} / \mathrm{min}$ was used in this step. To avoid air entrapment in the charcoal bed and reduce the formation of preferential channels, the system was operated in an up-flow regime.

\section{FOS purification}

Activated charcoal column was loaded with $455 \mathrm{~mL}$ of fermentative broth at a flow rate of $25 \mathrm{~mL} / \mathrm{min}$. The first $200 \mathrm{~mL}$ of the outlet stream was discarded. The broth was re-circulated until the equilibrium between the sorbent and the moving phase was obtained. Samples were collected from the column outlet for sugars quantification by HPLC.

To remove the nonadsorbed sugars, $7 \mathrm{~L}$ of Milli-Q pure water was passed through the column. Samples were continuously withdrawn from the column outlet and analyzed by HPLC to guarantee that all the nonadsorbed sugars were removed. The retained sugars were then recovered by elution with a gradient of ethanol (up to $50 \%(\mathrm{v} / \mathrm{v}))$. All experiments were performed at $25^{\circ} \mathrm{C}$.

Fractions collected in the desorption phase were evaporated at $60{ }^{\circ} \mathrm{C}$, to remove ethanol and concentrate sugars using a rotavapor (B. Braun Biotech International), and further freeze-dried (Heidolph lyophilizer).

The sodium, magnesium and potassium ions concentration was determined by flame atomic absorption spectrophotometry (Varian Spectra AA-400) in the final fractions and compared with the initial fermentative broth.

\section{Sugars analysis}

Samples were analyzed by HPLC using a Jasco device equipped with a refractive index detector and a Prevail Carbohydrate ES $(5 \mu \mathrm{m}, 250 \times 4.6 \mathrm{~mm})$ (Alltech). The mobile phase consisted of acetonitrile (HPLC grade; Carlo Erba) and water (70:30, v/v), with $0.04 \%$ of ammonium hydroxide in the water (HPLC grade; Sigma). Elution was conducted at $1 \mathrm{~mL} / \mathrm{min}$ flow rate and at room temperature $[24,25]$. 
FOS standards to determine the calibration curves were purchased from Wako, Chemicals GmbH (Germany). Sucrose, fructose and glucose (analytical grade) were obtained from Merck.

\section{Adsorption kinetic models}

Different kinetic models such as pseudo-first order [26] and pseudo-second order [27] have been used to predict the mechanism involved in the adsorption processes. In the present study the application of the pseudo-first and pseudo-second order models were tested for the sorption of FOS from a fermentative broth onto activated charcoal.

The pseudo-first order model is defined according to the following equation:

$\frac{d q_{t}}{d t}=k_{1}\left(q_{1}-q_{t}\right)$

where $q_{t}$ and $q_{1}$ are the amount of sugar adsorbed at time $t$ and equilibrium, respectively, and $k_{1}$ is the pseudo-first order rate constant.

The pseudo-second order equation is defined by the following equation:

$\frac{d q_{t}}{d t}=k_{2}\left(q_{2}-q_{t}\right)^{2}$

where $q_{2}$ is the amount of sugar adsorbed at equilibrium and $k_{2}$ is the pseudo-second order rate constant.

\section{Results and discussion}

\section{FOS production}

The sugars profile obtained in the fermentation of sucrose into FOS is illustrated in Fig. 1. Operation parameters were previously optimized to obtain the maximum production yield of FOS (unpublished data). A mass yield of $56 \%(\mathrm{w} / \mathrm{w})$ of FOS in total sugars was achieved at 50 hours of fermentation, having $44 \%$ $(\mathrm{w} / \mathrm{w})$ of glucose, fructose and sucrose in the mixture, which is in accordance with the results from Sangeetha and co-workers [4]. These authors reported a yield up to $57 \%(\mathrm{w} / \mathrm{w})$ of FOS using a culture broth homogenate of Aureobasidium sp.

However, it is important to notice that when the maximum yield is reached, the last sugar being produced, $\mathrm{GF}_{4}$, is usually

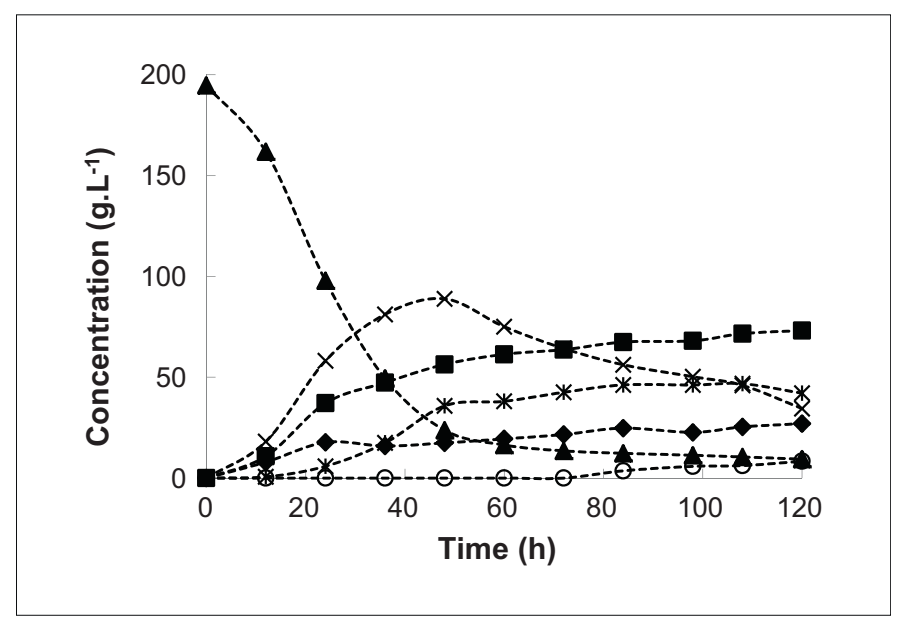

FIGURE 1

FOS production by Aureobasidium sp. Symbols: $\boldsymbol{\bullet}$, fructose; $\boldsymbol{\square}$, glucose; $\boldsymbol{\Delta}$ sucrose, $\times, \mathrm{GF}_{2} ; *, \mathrm{GF}_{3}$; and $\bigcirc, \mathrm{GF}_{4}$.

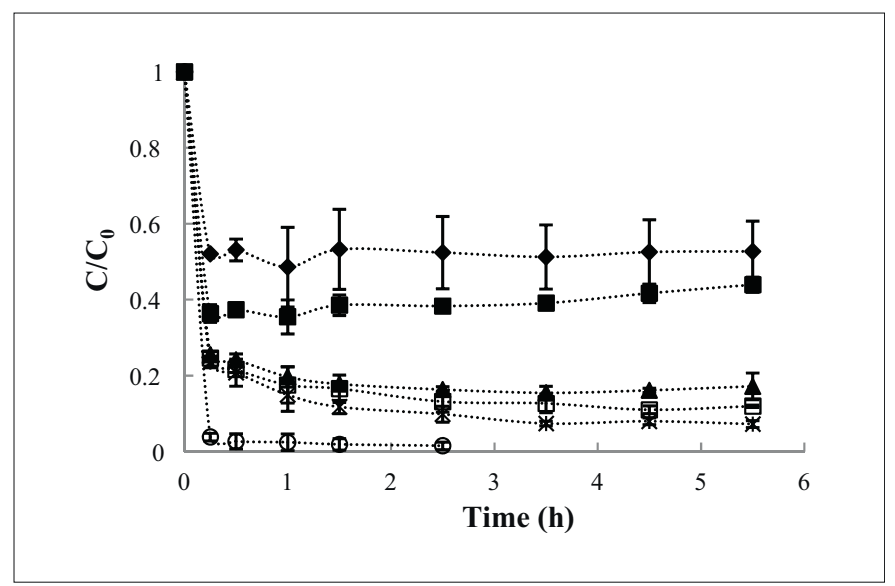

FIGURE 2

Adsorption profile of $\boldsymbol{\bullet}$, fructose; $\boldsymbol{\square}$, glucose; $\mathbf{\Delta}$, sucrose; $\square, \mathrm{GF}_{2} ; *, \mathrm{GF}_{3}$; and $\bigcirc, \mathrm{GF}_{4}$ from a fermentative broth, adsorbed on activated charcoal, in a shaken flask at $25^{\circ} \mathrm{C}$ and $140 \mathrm{rpm}$.

present in residual amounts. Therefore, as a process for the purification of the whole FOS mixture is envisaged, the fermentative broth was collected after this point and before $\mathrm{GF}_{2}$ starts to be degraded with consequent production of high contents of glucose. On the basis of this, the fermentation was stopped at 98 hours of operation and the respective fermentative broth was collected for further purification.

\section{Adsorption}

\section{Adsorption assays}

To evaluate the activated charcoal potential as an adsorbent for the purification of FOS from a fermentative broth mixture, equilibrium and adsorption kinetic studies were conducted in shaken flasks.

Adsorption curves demonstrated that sugars are selectively adsorbed according to their molecular weights (Fig. 2). Therefore, the sugar retention increased in the following order: $\mathrm{GF}_{4}>\mathrm{GF}_{3}>$ $\mathrm{GF}_{2}>\mathrm{S}>\mathrm{G}>\mathrm{F}$. It is important to notice that in the case of $\mathrm{GF}_{4}$ the levels of equilibrium concentration were very low to be accurately determined in the last sampling time points.

The adsorption behavior of each adsorbate (in this case each sugar) to a specific activated charcoal depends on the micropores diameter distribution of the granular activated charcoal [28]. The strength of the interaction between the adsorbate and activated charcoal increases with increasing molecular weight and size of the adsorbate molecules. According to Hung and co-workers [29] an adsorption dependence on the molecular weight and size occurs for highly agitated batch reactors, such as the system used in the current work. Therefore, it is expected that oligosaccharides adsorb more than other small saccharides (e.g. glucose or fructose) present in a given mixture.

In addition, a major portion of the carbon surface of the activated charcoal is nonpolar or hydrophobic [29]. Accordingly, the more hydrophobic the analyte is, the more retained will be in the charcoal [30]. Moreover, the hydrophobic character of the sugar is related with the extent of the $\mathrm{CH}$ groups surface area present in the saccharide structure [31]. Therefore, as oligosaccharides have an increased chain of $\mathrm{CH}$ groups, it is probably that they 

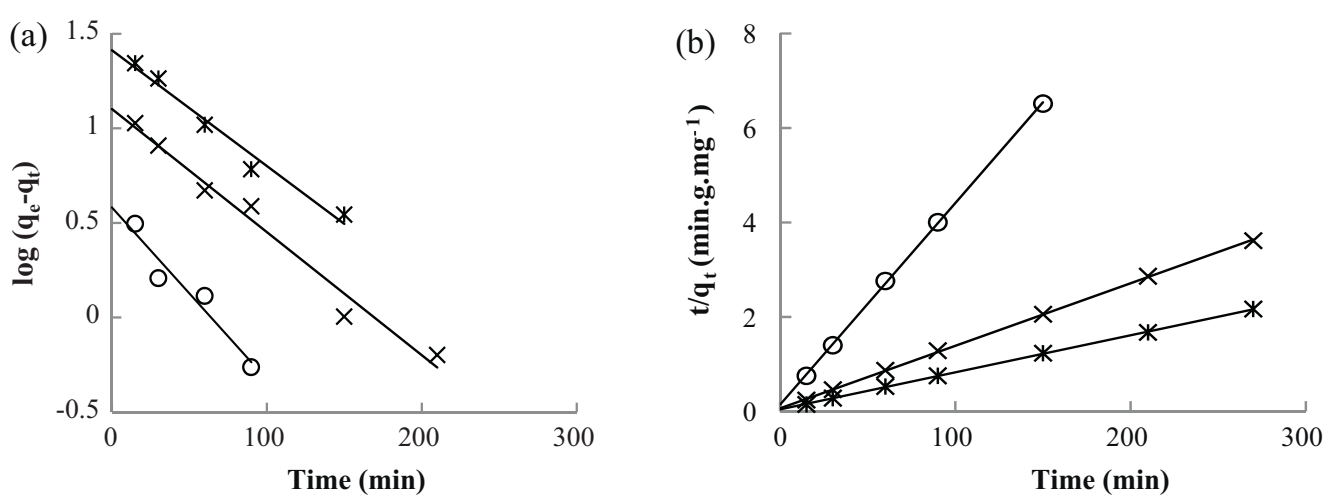

\section{FIGURE 3}

Linearized data of (a) the pseudo-first-order kinetic model and (b) the pseudo-second-order kinetic model for $\times, \mathrm{GF}_{2} ; *, \mathrm{GF}_{3} ;$ and $\bigcirc, \mathrm{GF}_{4}$ adsorption onto activated charcoal.

are more adsorbed to the activated charcoal than other small saccharides.

The type, position and orientation of the functional groups that contact with the adsorbent are also relevant for the retention process [32]. Although glucose and fructose have the same molecular weight, glucose was found to adsorb more onto activated charcoal than fructose, which may be related with the accessibility of the $\mathrm{CH}$ groups of glucose [31]. Accordingly, other authors also reported a higher adsorption of glucose in comparison to fructose $[14,30]$. However, it is important to note that glucose is present in higher concentrations in the fermentative broth than fructose, and charcoal is known to retain according to the amount of adsorbent in water; thus, glucose is expected to be more adsorbed than fructose.

In the current work, the adsorption process was very fast and the equilibrium was reached for the monosaccharides during the first 15 minutes. The remaining saccharides present in the mixture reached the equilibrium after around 2 hours of contact. Therefore, for the following studies conducted in the activated charcoal column, a 3 hours contact time was selected. For the shaken flask experiments, the equilibrium loading (q) calculated for FOS and mono- with disaccharides adsorbed onto activated charcoal was 223 and $150 \mathrm{mg}$, respectively, per g of activated charcoal.

\section{Adsorption kinetic models}

Plots representing the linearized first-order and second-order model equations (Eqs. (2) and (3)) are shown in Fig. 3. Values of the adsorption rates $-k_{1}$ and $k_{2}$ - and the respective loading equilibrium $-q_{1}$ and $q_{2}$ - were calculated from the slopes and intercepts of the linearized models (Table 1).
From Table 1 it can be seen that generally the $q$ values are greater for $\mathrm{GF}_{3}$ as compared to the other FOS. If all the sugars were present at the same concentration it would be probably that $\mathrm{GF}_{4}$ would be the most adsorbed based on its molecular weight, as previously mentioned. However, the fermentative broth contains distinct concentrations of each FOS. $\mathrm{GF}_{4}$ had a much lower concentration as compared to the other FOS. $\mathrm{GF}_{2}$ and $\mathrm{GF}_{3}$ were present at similar concentrations, thus similar $q$ values would be expected. Nevertheless, $\mathrm{GF}_{3}$ shows a greater $q$ value probably due to a combined effect of concentration and molecular weight, thus justifying the differences.

The pseudo-second order model seems to better describe the FOS adsorption mechanism because the coefficients of determination $\left(R^{2}\right)$ obtained are higher compared to the ones obtained for the pseudo-first order model. Moreover, the pseudo-second order model predicted the adsorption behavior for the whole range of contact time studied, while the pseudo-first order model was only applicable for the initial contact time. Accordingly, Ho and McKay [33] reported that in most cases the pseudo-first order model is generally applicable only for the initial contact time.

Finally, the pseudo-second order model was found to describe perfectly the adsorption of FOS onto activated charcoal as the $R^{2}$ values for all sugars are very high and the equilibrium capacities, $q_{2}$, determined by the model, are in agreement with the experimental $q_{e}$ values.

\section{FOS purification}

Several experiments were previously conducted to determine the best operating parameters for purifying FOS using a fixed bed activated charcoal column (data not shown). Parameters to be used

\section{TABLE 1}

Coefficients of determination of the linearized pseudo-first and pseudo-second order models and the respective modeled and experimental parameters

\begin{tabular}{|c|c|c|c|c|c|c|c|}
\hline \multirow[t]{2}{*}{ Sugar } & \multicolumn{3}{|c|}{ Pseudo-first order } & \multicolumn{3}{|c|}{ Pseudo-second order } & \multirow{2}{*}{$\begin{array}{l}\text { Experimental } \\
q_{\mathrm{e}}\left(\mathrm{mg} \mathrm{g}^{-1}\right)\end{array}$} \\
\hline & $q_{1}\left(\mathrm{mg} \mathrm{g}^{-1}\right)$ & $k_{1}\left(\min ^{-1}\right)$ & $R^{2}$ & $q_{2}\left(\mathrm{mg} \mathrm{g}^{-1}\right)$ & $k_{2}\left(g \mathrm{mg}^{-1} \min ^{-1}\right)$ & $R^{2}$ & \\
\hline $\mathbf{G F}_{2}$ & 12.7 & $1.50 \mathrm{E}-02$ & 0.978 & 75.8 & $2.68 \mathrm{E}-03$ & 1.000 & 74.0 \\
\hline $\mathrm{GF}_{3}$ & 25.9 & $1.40 \mathrm{E}-02$ & 0.975 & 126.6 & $1.53 \mathrm{E}-03$ & 0.999 & 125.6 \\
\hline $\mathrm{GF}_{4}$ & 3.8 & $2.10 \mathrm{E}-02$ & 0.934 & 23.4 & $1.28 \mathrm{E}-02$ & 1.000 & 23.0 \\
\hline
\end{tabular}




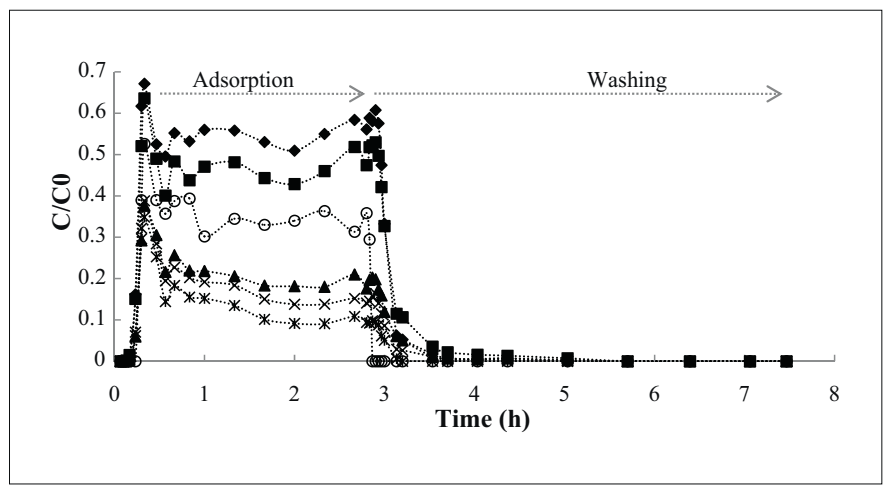

FIGURE 4

Adsorption and desorption kinetics of $\bullet$, fructose; $\boldsymbol{\square}$, glucose; $\boldsymbol{\Delta}$, sucrose; $\times$, $\mathrm{GF}_{2} ; *, \mathrm{GF}_{3} ;$ and $\mathrm{O}_{1} \mathrm{GF}_{4}$ from a fermentative broth on activated charcoal in a fixed column during the recirculation and washing phases (steps 1 and 2).

in the process, such as flow rate, recirculation time, water volume, ethanol volume and percentage, were rigorously studied.

Because the adsorption process is exothermic [34], the use of high temperatures can reduce the degree of adsorption. On the basis of that, and envisaging a lower impact on the process costs, a $25{ }^{\circ} \mathrm{C}$ room temperature was used in the current work.

\section{Column adsorption (step 1)}

After conditioning the column, the charcoal was loaded with sugars by recirculation of the fermentative broth (see section 'Activated charcoal column'). To avoid dilution of the broth, the first $200 \mathrm{~mL}$ of the outlet stream containing the water from the dead volume and the first eluted sugars in very low concentrations, were discarded. In this step, 3.9\% (w/w) of the FOS present in the initial mixture was lost.

The broth was kept in recirculation until the equilibrium between the concentration of sugars in the liquid and solid phases was reached. As shown in Fig. 4 the ratio $\mathrm{C} / \mathrm{CO}$ stabilizes after 1.5 hours of contact time. These results are in accordance with the ones previously obtained in the shaken flask experiments (adsorption assays). However, to assure that the maximum amount of sugars was adsorbed during the loading phase, the fermentative broth was kept in recirculation during 3 hours.

The ratios $C / C_{0}$ obtained in the adsorption phase, at the equilibrium, were $0.55 \pm 0.02$ for F; $0.46 \pm 0.03$ for $\mathrm{G}$; $0.19 \pm 0.01$ for $S$; $0.14 \pm 0.01$ for $\mathrm{GF}_{2} ; 0.10 \pm 0.01$ for $\mathrm{GF}_{3}$; and $0.34 \pm 0.02$ for $\mathrm{GF}_{4}$. As expected, the $C / C_{0}$ ratio increased as the molecular weight of the sugar decreased, except for $\mathrm{GF}_{4}$. The amount of $\mathrm{GF}_{4}$ in the fermentative broth was very low $\left(C_{0}=4.16 \mathrm{~g} \mathrm{~L}^{-1}\right)$, thus the concentration in the equilibrium was also very low and difficult to measure. Therefore, the $C$ calculated for $\mathrm{GF}_{4}$ may have been overestimated leading to the high $C / C_{0}$ ratios observed.

Results show that the least adsorbed sugars from the fermentative broth were fructose and glucose. Only 53.2\% and 62.5\% (w/w) of the fructose and glucose contained in the initial broth were

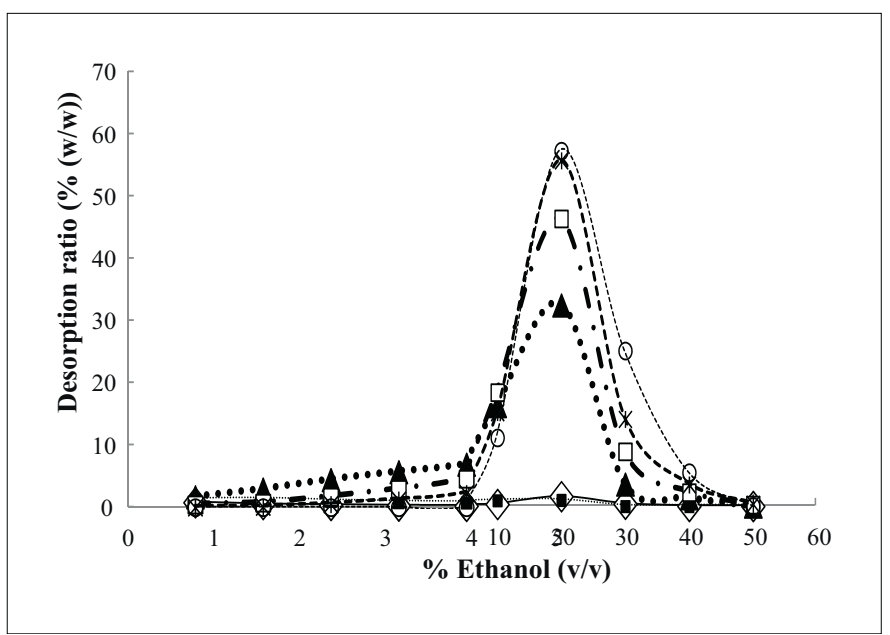

FIGURE 5

Desorption ratio of sugars ( $\diamond$, fructose; $\mathbf{\square}$, glucose; $\boldsymbol{\Lambda}$, sucrose; $\square, \mathrm{GF}_{2} ; *$, $\mathrm{GF}_{3}$, and $\mathrm{O}, \mathrm{GF}_{4}$ ) collected in each fraction.

adsorbed. Furthermore, from the fermentative broth, $83.9 \%$ (S), 86.9\% $\left(\mathrm{GF}_{2}\right)$, 90.4\% $\left(\mathrm{GF}_{3}\right)$ and $95.1 \%\left(\mathrm{GF}_{4}\right)(\mathrm{w} / \mathrm{w})$ were adsorbed onto the activated charcoal column. In summary, only $24 \%(\mathrm{w} / \mathrm{w})$ of the total sugars contained in the fermentative broth were not adsorbed and were initially discarded. Also, 77\% (w/w) of them constitute the small sugars (mono- and disaccharides) that should be eliminated in a FOS purification process. Losses of $7.0 \%(\mathrm{w} / \mathrm{w})$ of FOS from the fermentative broth were estimated in the adsorption step, namely in the recirculation.

The equilibrium loading $(q)$ determined on the column assay was $222 \mathrm{mg}$ of FOS per $\mathrm{g}$ of activated charcoal which is in accordance with the results obtained previously on the shaken flask experiments (adsorption assays).

\section{Column washing (step 2)}

A washing stage was included in the procedure to guarantee that all the nonadsorbed sugars were removed and the adsorbed monosaccharides were eluted. Therefore, the washing stage was carried out until no sugar was detected in the mobile phase. During this step, the outlet concentration was monitored by HPLC (Fig. 4). After 2 hours of washing, the outlet concentration indicated that the liquid phase was free of sugars. Furthermore, to assure a total cleansing, the washing continued for an extra 2 hours.

In this step, the desorption ratio representing the amount of sugar desorbed per adsorbed sugar was: 96\% (F), 93\% (G), 25\% (S), $13 \%\left(\mathrm{GF}_{2}\right), 7 \%\left(\mathrm{GF}_{3}\right)$ and $1 \%\left(\mathrm{GF}_{4}\right)(\mathrm{w} / \mathrm{w})$. Almost all the adsorbed monosacharides (step 1) were removed during the washing step. Moreover, FOS losses were estimated to be only $8.4 \%$ (w/w).

\section{Desorption (step 3)}

As previously demonstrated by the kinetic studies (see section 'Adsorption') and the adsorption onto the column (see section 'Column adsorption (step 1)'), sugars were selectively adsorbed

TABLE 2

Volume and ethanol concentrations used in step 3 to recover the sugars adsorbed onto the activated charcoal column

\begin{tabular}{|c|c|c|c|c|c|c|c|c|c|c|}
\hline$\%$ Ethanol (v/v) & 1 & 2 & 3 & 4 & 5 & 10 & 20 & 30 & 40 & 50 \\
\hline Volume of elution (L) & 0.5 & 0.5 & 0.5 & 0.5 & 0.5 & 1 & 2 & 2 & 1 & 0.5 \\
\hline
\end{tabular}




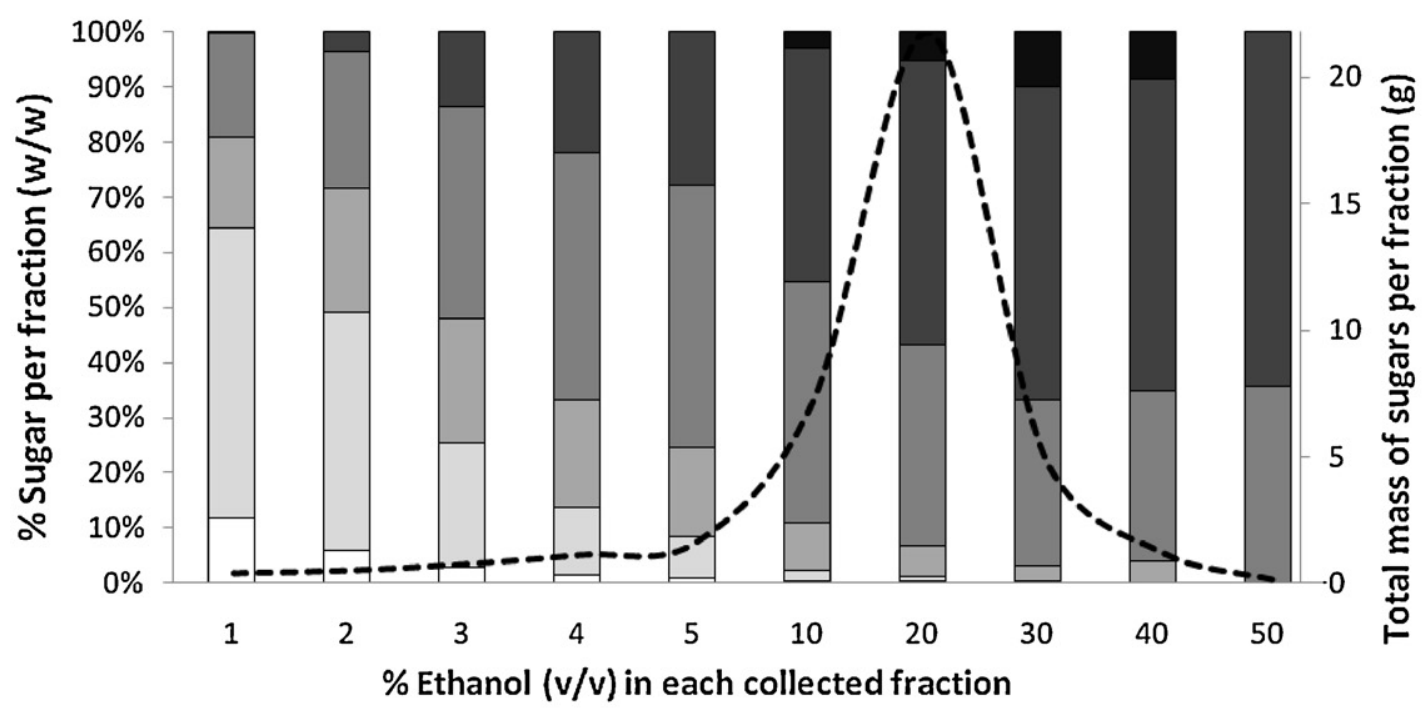

\section{FIGURE 6}

Recovery sugars per fraction desorbed $(-)$ and respective percentage of sugar ( $\square$, fructose; , glucose;, sucrose;, $\left.\mathrm{GF}_{2} ;, \mathrm{GF}_{3} ; \square, \mathrm{GF}_{4}\right)$ in each collected fraction.

onto activated charcoal. FOS were adsorbed more strongly than mono- and disaccharides. Thus, an adequate desorption strength should be used to selectively recover the sugars.

Several authors used water or very small percentages of ethanol to recover monosaccharides. As for disaccharides recovery, percentages between 5 and $10 \%$ of ethanol in water have been used. Oligosaccharides have been recovered using 15-50\% of ethanol in water $[14,17,18,22,23,35]$. Therefore, for the volume and percentage of ethanol/water used in each step, the desorption process was critically evaluated. Taking into account previous (data not shown) and current data, the optimized volume and percentage of ethanol were established for the desorption process (Table 2).

Because FOS are only desorbed when ethanol concentrations are between 10 and $40 \%(\mathrm{v} / \mathrm{v})$, a greater ethanol volume was used during the elution at these concentrations to recover all adsorbed FOS. The ethanol concentration that yielded the highest amount of sugars recovery was $20 \%(\mathrm{v} / \mathrm{v})$. Nonoligosaccharides need a lower strength to desorb; thus, a lower gradual increase of ethanol concentrations was used, up to $5 \%(\mathrm{v} / \mathrm{v})$. The gradient allowed the removal of more small saccharides at low ethanol concentrations, thus reducing the loss of FOS at the highest ones. In the end of the desorption step, and to assure that all sugars were desorbed, a 50\% $(\mathrm{v} / \mathrm{v})$ ethanol concentration was used.

Figure 5 shows the desorption ratio of each sugar, representing the percentage of sugar desorbed in step 3 per sugar adsorbed in step 1 , and the corresponding composition of the ethanol solution used to collect each fraction.

Figure 6 illustrates the amount of sugars recovered in each fraction (total mass of sugars per fraction), and the corresponding individual sugar percent composition $(\%, w / w)$. From both figures (Figs 5 and 6), a peak can be observed during the desorption step showing that ethanol efficiently desorbs the sugars retained in the column. $S$ and the remaining monosacharides ( $F$ and $G$ ) were desorbed at ethanol percentages below $5 \%(\mathrm{v} / \mathrm{v})$. The fraction that yielded the highest percentage of FOS $(97 \%(w / w))$ was eluted with
$30 \%(\mathrm{v} / \mathrm{v})$ of ethanol. Fractions rich in FOS were obtained with ethanol percentages between 10 and $40 \%(\mathrm{v} / \mathrm{v})$. The purity of FOS in these fractions was found to be $92.9 \%(\mathrm{w} / \mathrm{w})$ representing $74.5 \%$ $(\mathrm{w} / \mathrm{w})$ of FOS from the fed fermentative broth. Furthermore, $32.7 \mathrm{~g}$ of FOS could be recovered from the initial mixture (containing $43.9 \mathrm{~g}$ of FOS) fed to the activated charcoal column.

Through the analysis of the desorption ratios it was also possible to observe that $\mathrm{S}, \mathrm{GF}_{2}, \mathrm{GF}_{3}$ and $\mathrm{GF}_{4}$ were preferentially desorbed with 5, 10, 20 and 30\% ethanol, respectively.

\section{Desalting}

As previously discussed, the culture medium used for the microbial production of FOS contains high amounts of salt. Consequently, in the end of the fermentation process many ions are present in the solution. Desalting the fermentative broth constitutes an important step to guarantee certain quality parameters. Therefore as sodium, potassium and magnesium are the main cations present in the fermentation culture medium, their amounts were determined in the recovered pure FOS fractions. Results showed that these pure FOS fractions were free of ions, confirming the effectiveness of activated charcoal in the desalting of the fermentative broth, which is in accordance with several studies that have been reported $[13,14]$.

\section{Conclusion}

Adsorption kinetic experiments show that sugars were selectively adsorbed and that FOS adsorption onto activated charcoal was well described by a pseudo-second order model.

The fermentation of sucrose by Aureobasidium sp. to produce FOS resulted in a sugar mixture containing $56 \%(\mathrm{w} / \mathrm{w})$ of FOS. This fermentative broth was further purified using an activated charcoal column. After the optimization of the ethanol volume and concentration used in the global desorption step, ethanol solutions ranging from 10 to $40 \%(\mathrm{v} / \mathrm{v})$ were used to recover FOS. These ethanol solutions enabled a FOS recovery of $74.5 \%(\mathrm{w} / \mathrm{w})$ with a 
92.9\% (w/w) of purity, thus leading to a FOS product with a higher market value. Moreover, with the proposed process, fractions were recovered with purities up to $97 \%$ (w/w) of FOS. Activated charcoal was also found to be very efficient in the desalting of the fermentative broth.

In summary, this work described a simple and efficient process to purify FOS from a fermentative broth using a single column filled with commercial activated charcoal that can be easily applied at a larger scale.

\section{Acknowledgement}

The first author gratefully acknowledges to Fundação para a Ciência e a Tecnologia (Portugal) for the PhD Grant received (reference SFRH/BD/32514/2006).

\section{References}

1 Kolida, S. and Gibson, G.R. (2007) Prebiotic capacity of inulin-type fructans. J. Nutr. 137, 2503S-2506S

2 Gibson, G.R. (1998) Dietary modulation of the human gut microflora using prebiotics. Br. J. Nutr. 80, S209-S212

3 Sangeetha, P.T. et al. (2005) Recent trends in the microbial production, analysis and application of fructooligosaccharides. Trends Food Sci. Technol. 16, 442-457

4 Sangeetha, P.T. et al. (2004) Production of fructo-oligosaccharides by fructosyl transferase from Aspergillus oryzae CFR 202 and Aureobasidium pullulans CFR 77. Process Biochem. 39, 753-758

5 Sanz, M.L. and Martinez-Castro, I. (2007) Recent developments in sample preparation for chromatographic analysis of carbohydrates. J Chromatogr. A 1153 74-89

6 Botelho-Cunha, V.A. et al. (2010) Tailoring the enzymatic synthesis and nanofiltration fractionation of galacto-oligosaccharides. Biochem. Eng. J. 50, 29-36

7 Goulas, A.K. et al. (2003) Fractionation of oligosaccharides by nanofiltration. J. Sci. Food Agric. 83, 675-680

8 Li, W.Y. et al. (2004) Study on nanotiltration for purifying fructo-oligosaccharides: I. Operation modes. J. Membr. Sci. 245, 123-129

$9 \mathrm{Ku}$, Y. et al. (2003) Precipitation of inulins and oligoglucoses by ethanol and other solvents. Food Chem. 81, 125-132

10 Kuhn, R.C. and Maugeri, F. (2010) Purification of fructoligosaccharides in active carbon fixed-bed column. New Biotechnol. 25, S182

11 Hernandez, O. et al. (2009) Comparison of fractionation techniques to obtain prebiotic galactooligosaccharides. Int. Dairy J. 19, 531-536

12 Chinn, D. and King, C.J. (1999) Adsorption of glycols, sugars, and related multiple -OH compounds onto activated carbons. 2. Solvent regeneration. Ind. Eng. Chem. Res. 38, 3746-3753

13 Packer, N.H. et al. (1998) A general approach to desalting oligosaccharides released from glycoproteins. Glycoconj. J. 15, 737-747

14 Whistler, R.L. and Durso, D.F. (1950) Chromatographic separation of sugars on charcoal. J. Am. Chem. Soc. 72, 677-679

15 Capek, K. and Stanek, J., Jr (1975) Carbohydrates. In Journal of Chromatography Library, Liquid Column Chromatography A Survey of Modem Techniques and Applications (Deyl, Z., Macek, K., Janak, J., eds), pp. 465-522, Elsevier

16 Weston, R.J. and Brocklebank, L.K. (1999) The oligosaccharide composition of some New Zealand honeys. Food Chem. 64, 33-37

17 Morales, V. et al. (2006) Rapid separation on activated charcoal of high oligosaccharides in honey. Chromatographia 64, 233-238

18 Swallow, K.W. and Low, N.H. (1990) Analysis and quantitation of the carbohydrates in honey using high-performance liquid-chromatography. J. Agric. Food Chem. 38, 1828-1832
19 Priem, B. et al. (2002) A new fermentation process allows large-scale production of human milk oligosaccharides by metabolically engineered bacteria. Glycobiology $12,235-240$

20 Boon, M.A. et al. (2000) Enzymatic synthesis of oligosaccharides: product removal during a kinetically controlled reaction. Biotechnol. Bioeng. 70, 411-420

21 Ajisaka, K. et al. (1987) Use of an activated carbon column for the synthesis of disaccharides by use of a reversed hydrolysis activity of beta-galactosidase. Biotechnol. Lett. 9, 387-392

22 Hidaka, H. et al. (1988) A fructooligosaccharide-producing enzyme from Aspergillus-Niger Atcc-20611. Agric. Biol. Chem. 52, 1181-1187

23 Kaplan, H. and Hutkins, R.W. (2000) Fermentation of fructooligosaccharides by lactic acid bacteria and bifidobacteria. Appl. Environ. Microbiol. 66, 2682-2684

24 Dias, L.G. et al. (2009) UV spectrophotometry method for the monitoring of galacto-oligosaccharides production. Food Chem. 113, 246-252

25 Nobre, C. et al. (2009) Comparison of adsorption equilibrium of fructose, glucose and sucrose on potassium gel-type and macroporous sodium ion-exchange resins. Anal. Chim. Acta 654, 71-76

26 Lagergren, S. (1898) About the theory of so-called adsorption of soluble substances. Kungliga Svenska Vetenskapsakademiens Handlingar 24, 1-39

27 Ho, Y.S. and Mckay, G. (1999) Pseudo-second order model for sorption processes. Process Biochem. 34, 451-465

28 Hidaka, H., et al. (1981) Method of producing high purity maltose. USP4294623

29 Hung, Y.T. et al. (2005) Granular activated carbon adsorption. In Physicochemical Treatment Processes (Wang, L.K., Hung, Y.T., Shammas, N.K., eds), pp. 573-633, Humana Press

30 Abe, I. et al. (1983) Adsorption of saccharides from aqueous solution onto activated carbon. Carbon 21, 189-191

31 Sundari, C.S. and Balasubramanian, D. (1997) Hydrophobic surfaces in saccharide chains. Prog. Biophys. Mol. Biol. 67, 183-216

32 Koizumi, K. (1996) High-performance liquid chromatographic separation of carbohydrates on graphitized carbon columns. J. Chromatogr. A 720, 119-126

33 Ho, Y.S. and Mckay, G. (1998) A comparison of chemisorption kinetic models applied to pollutant removal on various sorbents. Process Saf. Environ. Protect. 76, 332-340

34 Inglezakis, V.J. and Poulopoulos, S.G. (2006) Adsorption and Ion Exchange. Adsorption, Ion Exchange and Catalysis, Design of Operations and Environmental Applications. Elsevier pp. 243-353

35 Sanz, M.L. et al. (2005) In vitro investigation into the potential prebiotic activity of honey oligosaccharides. J. Agric. Food Chem. 53, 2914-2921 